\title{
Justicia y racionamiento sanitario en el Plan AUGE: dilemas bioéticos asociados a la distribución de recursos escasos
}

\author{
Alejandra Zúñiga Fajuri ${ }^{1}$
}

Resumen: Los criterios de racionamiento en materia sanitaria establecidos por el Plan AUGE en Chile, se fundan principalmente en máximas de eficiencia que son contrarias a los principios de equidad que debieran sustentar las políticas sanitarias de las sociedades democráticas. Para la aplicación y justificación de las técnicas de racionamiento sanitario se requiere no sólo una reflexión desde la ciencia médica y la economía de la salud, sino que también desde la filosofía moral y la bioética. El estudio de las teorías de la justicia distributiva sanitaria permite valorar críticamente los criterios de racionamiento sanitarios utilizados por las Guías Clínicas de cada una de las patologías GES/AUGE que resultan contrarios al principio de equidad.

Palabras clave: cuidado sanitario, racionamiento sanitario, equidad, eficiencia

\section{Justice and health care rationing in AUGE: bioethical dilemmas associated to the distribution of scarce resources}

\begin{abstract}
Rationing criteria in health care establish by AUGE, in Chile, are based, mainly, in maximum efficiency, contrary to the equity principle, which should sustain health care policies in democratic societies. For the application and justification of health care rationing techniques is required not only a medical science and health care economy reflection, but a moral philosophy and bioethical reflection as well. The study of health care distributive justice theories allows to value critically health care rationing criteria used by Clinical Guidelines for each GES/AUGE pathology, which operate contrary to the equity principle.
\end{abstract}

Key words: health care, health care rationing, equity, efficiency

\section{Justiça e fundamentação sanitária no Plano AUGE: dilemas bioéticos associados à distribuiçáo de recursos escassos}

Resumo: Os critérios de fundamentação em matéria sanitária estabelecidos pelo Plano AUGE, no Chile, se fundam, principalmente, em máximas de eficiência que são contrárias aos princípios de equidade que deveriam sustentar as políticas sanitárias ds sociedades democráticas. Para a aplicação e justificação das técnicas de racionamento sanitário se requer não só uma reflexão a partir da ciência médica e da economia da saúde, mas, também, a partir da filosofia moral e da bioética. O estudo das teorias da justiça distributiva sanitária permite valorar criticamente os critérios de racionamento sanitário utilizados pelas Guias Clínicas de cada uma das patologias GES/AUGE que resultam contrários ao princípio da equidade.

Palavras-chave: cuidado sanitário, racionamento sanitário, equidade, eficiencia

\footnotetext{
${ }^{1}$ Doctora en Derecho. Profesora de la Escuela de Derecho de la Universidad de Valparaíso y de la Escuela de Derecho de la Universidad Diego Portales, Chile

Correspondencia: alejandra.zuniga@uv.cl
} 


\section{Introducción ${ }^{2}$}

Una correcta comprensión del derecho a la protección de la salud debe considerar los problemas relacionados con la distribución equitativa de los recursos sanitarios y su necesario racionamiento, generando respuestas a los principales problemas de justicia asociados, sobre la base de fundamentos teóricos que, desde la idea de derechos humanos e igualdad moral de las personas, justifiquen el uso de criterios de adjudicación compatibles con dichos principios. El tipo de modelo de asignación que se estime ético dependerá de la concepción democrática de persona moral que se sustente y de la teoría de la justicia distributiva que finalmente se defienda.

La presente investigación ha pretendido evaluar críticamente los criterios de racionamiento sanitarios utilizados por las Guías Clínicas de cada una de las Garantías Explícitas en Salud (GES) de la Reforma AUGE (Acceso Universal de Garantías Explícitas) en Chile, a través del estudio de las teorías de la justicia distributiva sanitaria más relevantes en el área de la bioética y sus propuestas de racionamiento de recursos escasos, fundadas en principios compatibles con las demandas de equidad, responsabilidad y eficiencia propias de las sociedades democráticas. La excesiva utilización de instrumentos de adjudicación propios de la economía de la salud al momento del establecimiento de las Guías Clínicas, sin atender a su carácter más o menos equitativo, demuestra la necesidad de reflexionar sobre la coherencia entre los sustratos teóricos que, en su momento, dieron cimiento y apoyo político a la Reforma y los resultados inequitativos de su ejecución.

El derecho al cuidado sanitario y la teoría de la justicia del liberalismo igualitario

Para lidiar con los problemas asociados a las desigualdades en el acceso a recursos básicos, todas las teorías de la justicia inician sus reflexiones desde un principio "formal" de equidad, atribuido a Aristóteles, que reclama tratar igual a los iguales y desigual a quienes son desiguales. Es un principio

\footnotetext{
${ }^{2}$ Este artículo forma parte del proyecto de investigación No 11080005 financiado por el Fondo Nacional de Desarrollo Científico y Tecnológico (FONDECYT), titulado "Teorías de la justicia y Reforma Sanitaria AUGE”.
}

formal porque no establece criterios o ámbitos concretos que nos permitan saber cuándo la situación de determinadas personas puede calificarse como 'igual', por lo que se trata de un principio que debe ir acompańado de otro de tipo "material" que especifique las características relevantes para el tratamiento igual. Así es como todas las políticas públicas de tipo distributivo se construyen sobre alguno de los siguientes criterios materiales de justicia:

- A cada cual una parte igual de los recursos sociales.

- A cada cual según su necesidad.

- A cada cual según su esfuerzo.

- A cada cual según su contribución social.

- A cada cual conforme con su mérito.

- A cada cual conforme con las reglas de intercambio libre del mercado(1).

¿Cuál o cuáles de estos criterios materiales de justicia son recogidos por la teoría de la justicia del liberalismo igualitario?

El liberalismo igualitario nace con la publicación de "Theory of justice" de John Rawls quien, con el objeto de construir un modelo de justicia que regule las instituciones sociales básicas, utiliza la teoría clásica del contrato social y del constructivismo ético kantiano(1) para, a través de un procedimiento racional que asegure la equidad de las partes contratantes, concluir principios de justicia capaces de definir y consolidar nuestros derechos básicos $(2,3)$.

Para Rawls, las desigualdades sociales y económicas — reguladas por el segundo principio de justicia - tienen que satisfacer dos requisitos para ser legítimas: a) deben estar vinculadas a cargos y posiciones abiertos a todos en condiciones de igualdad equitativa de oportunidades, y b) deben redundar en el mayor beneficio de los miembros menos aventajados de la sociedad, lo que se conoce como "principio de diferencia"(4), que se sustenta en la idea de que los mejor dotados no merecen el lugar que, por suerte, les ha tocado en la lotería natural y social(5). Ahora, si bien Rawls consideraba indeseables las desigualdades sociales, su capacidad para aumentar los grados de eficiencia productiva las harían necesarias, de modo que 
la injusticia consistirá "simplemente en las desigualdades que no benefician a todos" (2:62-65).

Hay, sin embargo, quienes oponen a esta tesis una concepción del valor de la igualdad basada en la idea de que es en sí mismo malo que algunos estén peor que otros, concepción que Derek Parfit ha denominado "teleológica", en contraposición con una visión "deóntica" de la igualdad(6). Sin embargo, el problema de una concepción de la igualdad de este tipo es que no logra responder a la llamada objeción de la "igualación por lo bajo" (levelling down objection) desarrollada por este autor. Para los igualitaristas estrictos "Es malo (...) que algunos sean videntes y otros ciegos. Por ello tenemos una razón moral para extraer un ojo de quienes ven y dárselo a los ciegos, conclusión que parece horrible" (6:210), pues reprueban toda desigualdad, incluso la que es beneficiosa para algunos, sin perjudicar a nadie(7).

Por ello, los igualitaristas deónticos prefieren defender sólo el logro, para todos por igual, de un mínimo de aquello que se considere especialmente valioso. La desigualdad per se no sería realmente mala(8). Esta forma de entender la igualdad, conocida como "prioritarismo", no se detiene en las diferencias de riqueza entre los individuos, sino que busca lograr que los peor situados alcancen progresivamente una mejor posición, pues considera que la obligación moral de beneficiar a este grupo es directamente proporcional a la magnitud de sus privaciones $(9,10)$. La pregunta que surge de inmediato frente a la propuesta prioritarista es: ¿cuánto podemos gastar en mejorar a los más desaventajados? No podemos olvidar que la igualdad tiene costos que debemos considerar cuando estamos en condiciones de escasez moderada, si no queremos desembocar en situaciones realmente injustas.

Resulta preferible defender, con Frankfurt y Crisp, una tercera versión de la igualdad denominada "suficientismo", que plantea que, alcanzado un cierto nivel de satisfacción de preferencias o bienestar, acaban nuestras obligaciones de acudir en beneficio de quienes están peor, de modo que "si todo el mundo tuviera suficiente, no tendría consecuencias morales el hecho de que unos ten- gan más que otros"(8). Algo similar defienden Powers y Faden en su particular versión del "suficientismo del bienestar” que, desde el análisis de seis distintas dimensiones del bienestar, coloca su atención en los resultados alcanzados por los individuos y, por lo mismo, en las desigualdades en los medios para alcanzar dicho bienestar(11).

Desde esta perspectiva suficientista, ¿cuál es el mínimo decente que debemos cubrir en materia sanitaria? Ronald Dworkin ha desarrollado una importante crítica al llamado principio del rescate, el cual sostiene que, como la salud y la vida son los mayores y más importantes bienes, es necesario gastar todo lo que se tenga en cuidado sanitario (12,13). Como alternativa, el autor plantea el principio del seguro prudente, en el que los individuos decidirán el contenido de un "mínimo sanitario" en un contexto ideal que les permitirá acordar un cuidado en condiciones dignas, pero no tratamientos muy costosos que limiten sus planes de vida $(13,14)$. De este modo, podría fundamentarse la aparición de un seguro de carácter universal y prestaciones mínimas similar al que existe en Canadá, el Reino Unido o España(15).

Norman Daniels, por su parte, propone un criterio que relaciona el acceso a cuidado sanitario con el principio de la justa igualdad de oportunidades, desarrollado por Rawls, y que destaca la singularidad de este recurso y su habilidad para permitir a las personas elegir un plan de vida razonable. Se trataría de un criterio compatible con distintos sistemas de cuidado sanitario y que permite caracterizar de "suficiente" a un sistema que logra alcanzar el objetivo de mantener, restaurar o compensar por la pérdida del funcionamiento normal de la especie $(16,17)^{3}$. La tesis de Daniels provee de argumentos para determinar qué debe ser incluido en la lista básica y, además, dispone que no debe haber obstáculos de ningún tipo (raciales, financieros, regionales, etc.) para acceder al contenido mínimo de cuidado sanitario, pues las desigualdades en las oportunidades de las personas no debieran ser toleradas por motivos econó-

\footnotetext{
${ }^{3}$ Daniels compara la importancia de la salud con la de la educación sosteniendo que, al igual como resulta necesario disminuir las diferencias de origen social que colocan a algunos en una situación de desventaja educacional inmerecida, se puede sostener que no se merecen las desventajas genéticas que determinan un mal estado de salud (1985:46-47).
} 
micos(18). De este modo, la noción de mínimo sanitario es consistente con el suficientismo, pues evita el llamado "principio fuerte de igual acceso", de acuerdo con el cual todos tendrían un derecho igual al mejor servicio sanitario disponible, lo que supondría agotar todos los recursos en cuidado sanitario, dejando sin atención otros bienes sociales también importantes.

\section{Racionamiento sanitario, economía de la salud y equidad}

La bioética está plagada de decisiones trágicas en las que cualquier elección sobre la distribución de recursos afecta de manera sustancial la vida de las personas. Se puede distinguir entre elecciones trágicas en las que el bien a repartir es inevitablemente escaso - como ocurre con los trasplantes de órganos- y elecciones trágicas en las que el bien a repartir es insuficiente porque es muy caro. En este último caso, lo que parecía un problema de elección trágica puede transformarse en un asunto de elección política, en el cual la responsabilidad social no es fácil de evadir, pues el sufrimiento que provoca la escasez ya no aparece como impuesto sino que como 'elegido' (19).

La respuesta para el racionamiento que se ha dado desde la teoría de la justicia utilitarista viene de la mano de la llamada "economía de la salud" que, con su objetivo de maximización de los recursos sanitarios, ha elaborado el criterio QALY (Quality-Adjusted-Life-Years $)^{4}$, cuya esencia radica en darle valor de ' 1 ' a la esperanza de vida saludable de un año y en restarle el valor de ' 1 ' a la esperanza de vida no saludable de un año, de modo que el valor preciso de la vida de una persona enferma disminuirá en la medida en que lo haga su calidad de vida. La fuerza del QALY descansa en la idea de que, dada la posibilidad de elección, cualquier persona racional preferirá una vida más breve, pero saludable, que una larga en condiciones de severa discapacidad o enfermedad(20). Los QALY permiten analizar cómo una acción médica aumenta la expectativa de vida del paciente y asegura, además, calidad de vida(21), enfatizando la relevancia de los criterios de conveniencia, efectividad y responsabilidad pública en la adjudica-

4 En castellano AVAC, Años de vida ajustados a calidad. ción de recursos escasos(22) y destacando el principio de eficiencia como crucial al momento de resolver los problemas asociados al racionamiento de recursos sanitarios $(23,24)$.

Entre las críticas éticas más importantes a este modelo destaca aquella que sostiene que, al asignar un lugar prioritario al principio de eficiencia, se violenta de varias maneras el principio de equidad, al permitir la discriminación de pacientes por razones de edad, sexo, raza, motivos económicos, entre otros, pues serían grupos sociales 'ineficientes'. Así, por ejemplo, la recuperación total de una cierta condición médica de los ancianos y las personas discapacitadas será siempre, 'ceteris paribus', más costosa en términos QALY que la recuperación total de esa misma condición de personas más jóvenes y sanas(25).

La alternativa distributiva al utilitarismo viene de la mano de la teoría de la justicia del "igualitarismo sanitario", fundada en el principio de la necesidad sanitaria como fuente de derechos. Sobre este principio se defiende un modelo de adjudicación que permita la determinación de un mínimo sanitario decente que se garantice de modo "universal", esto es, para todas las personas por igual. Para John Harris, es una injusticia profunda el ejercicio de valorar las vidas al que nos obliga el criterio QALY(26). El principio que debiera gobernar nuestras decisiones de adjudicación de recursos escasos debiera ser aquel que defiende que todas las personas merecen las mismas oportunidades de aprovechar el sistema sanitario público y que ese derecho no es proporcional ni a las posibilidades de beneficiarse del servicio, ni a la calidad (resultado) del beneficio, ni al tiempo que se espera que ese beneficio pueda ser gozado(27).

El criterio QALY discrimina por edad, ya que los años de vida que es posible esperar que una persona disfrute a consecuencia de un tratamiento disminuyen automáticamente cuanto mayor edad tiene el paciente. Además, se produce el efecto negativo llamado Double Jeopardy, pues los QALY empeoran la situación de los que ya están peor desde el punto de vista de la enfermedad. Por ejemplo, al elegir para un trasplante entre una persona con alguna discapacidad y otra sin disca- 
pacidad se elegiría siempre a la persona sin discapacidad(28). En fin, los QALY también discriminan por razones sociales y/o económicas debido a que los pacientes con enfermedades más baratas y aquellos con una mala calidad de vida, al no poder garantizar el éxito del tratamiento, serían, en general, dejados de lado 5 . Cuando se utilizan únicamente criterios de eficiencia para la racionalización de los recursos, la mayoría de las personas queda disconforme, pues los argumentos clásicos de los economistas parecen más los de quienes, de alguna manera, "saben el precio de todo y el valor de nada” (29).

\section{El racionamiento sanitario en el Plan AUGE}

En términos generales, podemos clasificar los criterios de adjudicación de recursos en criterios clínicos, en el cual el recurso se asigna a aquel con mejores oportunidades de recuperación o aquel cuyas necesidades médicas sean mayores; criterios sociales, relativos al valor social actual o contribución potencial del individuo; y criterios igualitarios, que fundados en el principio de igualdad prefieren dejar la selección al azar o, en general, a juicios relacionados con el tiempo(30). Entre estos últimos criterios destacan las listas de espera, es decir, la regla de que "el primero que llega es el primero en ser atendido". Las razones para preferir este mecanismo serían, primero, que el sacrificio que significa estar parado en una fila se considera como un generador de merecimiento. Segundo, la voluntad de hacer fila se estima como una medida de la necesidad del bien. Y tercero, el uso de la fila es un valioso contrapeso al filo penetrante del dinero para asignar bienes escasos(31). El uso de estos criterios impersonales estaría justificado, puesto que, si los recursos sociales son escasos y no existen mayores disparidades en la utilidad médica para los pacientes, entonces las consideraciones de oportunidad justa, de igual respeto e igual evaluación de las vidas justifica la práctica de hacer fila, una lotería o la distribución de recursos al azar(32).

5 Con frecuencia, los más pobres responden peor a los tratamientos médicos que los ricos con igual dolencia, debido a que estos últimos disfrutan de mejores condiciones sociales, ambientales, higiénicas e incluso culturales, que los hace competir favorablemente en la obtención de QALYs, es decir, en capacidad de beneficiarse del tratamiento (Puyol A. Ética, derechos y racionamiento sanitario. Doxa 1999; 22).
Pero no todos están de acuerdo con la utilización del azar para distribuir recursos. Para Calabresi y Bobbitt, el mecanismo de tratar igual a todas las personas encarna la más simple e inocente concepción de la igualdad. En su más pura forma, la lotería no da peso alguno ni a los intereses individuales ni a los sociales, a menos que consideremos que el interés social está en tratar a todos de la misma manera, sin atender a las consecuencias. Tampoco la lotería evita el problema de los costos pues, si bien no pone precio a los bienes trágicos — como lo hace el mercado—, ni selecciona descarnadamente a las víctimas o perdedores de la elección — como cuando el Estado prioriza pacientes o tratamientos-, hace algo igualmente costoso: "enfatiza nuestra incapacidad para defender como absolutos derechos como, por ejemplo, el derecho a la vida" (33).

En fin, más allá de que podamos defender o no la asignación de recursos escasos por medio de mecanismos aleatorios, en una segunda etapa de selección de pacientes nos toca ahora preguntarnos ¿cuál de estos criterios - y con qué razonesse han utilizado en las Guías Clínicas GES de la Reforma del AUGE en Chile? En la óptica de los QALY, lo cierto es que la mayoría de las Guías Clínicas manifiestan con claridad que buscan "otorgar una atención de salud cuyos resultados (...) generen beneficios por sobre los riesgos de una determinada intervención" (34) sobre la base de que el sistema sanitario se beneficia en su conjunto cuando las decisiones médicas se realizan "identificando las intervenciones más efectivas y en lo posible las mas costo/efectivas" (35).

Es necesario recordar que, desde su inicio, no obstante varios estimaron que se trataba de una reforma idónea para solucionar los problemas de equidad del sistema y aun cuando la Ley de Acceso Universal con Garantías Explícitas (AUGE) —Ley No 19.966 de 2004- prometió establecer un Régimen de Garantías en Salud que aseguraría que "toda persona residente en el territorio nacional, independiente de su edad, sexo, educación, etnia, preferencias sexuales o ingreso, pueda contar con un mecanismo de protección social que le dé acceso universal a una atención de salud adecuada y oportuna para enfrentar las situaciones 
de enfermedad"6, las Guías Clínicas restringieron discriminatoriamente el acceso con el fin de permitir a la autoridad aumentar el número de enfermedades cubiertas. Esto significa que no existe en Chile aquello que, según se ha revisado, constituye una demanda imprescindible desde la óptica de las teorías de la justicia y de organizaciones internacionales como la Organización Mundial de la Salud: un "mínimo sanitario decente universal" $(36)^{7}$.

Al momento de discutirse la Reforma Sanitaria, la OMS situaba a Chile en el lugar número 168 de 191 países en la dimensión denominada 'fairness', o justicia en el financiamiento(37). Entonces Chile era uno de los países de América que menos gastaba en salud (sólo U\$ 642 per cápita, es decir, un 5,8 del PIB) lo que explicaba y explica aún hoy sus notables falencias. Además, como país en vías de desarrollo que mantiene grandes desigualdades sociales, Chile es prueba fehaciente de la relación entre los resultados sanitarios de un país y el estatus socioeconómico de sus habitantes(38). Por ello resulta importante evaluar la magnitud de la incorporación en el Plan AUGE de elementos de racionamiento derivados de análisis económicos que limitan la inclusión de criterios de equidad vitales para paliar la injusticia inherente del sistema sanitario chileno.

La Reforma se ha implementado de manera paulatina desde 2005 a través de tres regímenes AUGE que han ido, cada uno, incorporando nuevas enfermedades GES con sus correspondientes Guías Clínicas. A pesar de los incumplimientos en materia de acceso, la autoridad ha continuado defendiendo la ampliación del Plan a nuevas patologías, lo que es preocupante si se tiene en cuenta que, según evidencia la tabla 1 , los mecanismos de adjudicación utilizados en muchas de ellas, propios de la economía de la salud, han disminuido considerablemente el número de personas potencialmente beneficiarias.

\footnotetext{
${ }^{6}$ Véase el mensaje presidencial del Proyecto de Ley que establece un Régimen de Garantías en Salud AUGE, de 22 de mayo de 2002. N ${ }^{\circ}$ Boletín: 2947-11. Lo destacado es mío.

${ }^{7}$ El objetivo de los Sistemas Nacionales de Salud es, para la OMS, disminuir las iniquidades sanitarias y el fenómeno que demuestra la estrecha relación entre pobreza y mala salud, "lo que significa que las personas que viven en la pobreza reciben menos de la parte proporcional que les corresponde de los fondos públicos para salud en comparación con las clases acomodadas". OMS; 2003: 136-137.
}

Como se puede apreciar, la incorporación de criterios de exclusión o limitación en el acceso relacionados con la edad del paciente o su lugar de residencia en prácticamente todas las patologías GES no responden adecuadamente a las promesas de equidad que fundamentaron la Reforma. De esta tabla se concluye que, de las 77 guías clínicas vigentes hasta la fecha, más del $70 \%$ dispone criterios de exclusión que violentan el principio de igualdad defendido por las teorías de la justicia tanto liberales igualitarias como igualitaristas.

Entre los casos en que los criterios de racionamiento del utilitarismo sanitario se aprecian con claridad destacan, primero, la GES de analgesia en el parto que sólo es accesible en la medida en que lo permitan "los recursos locales humanos y físicos" de la localidad en la que viva la embarazada, cuestión que, debido a la reducida cantidad de centros hospitalarios dotados de especialistas anestesistas, hace que el cumplimiento de dicha garantía sea extraordinariamente bajo(39). Por su parte, la Guía Clínica de Artrosis de Cadera excluye a las personas menores de 65 ańos, aun cuando reconoce que el $40 \%$ de las personas mayores de 60 años también padecen de artralgias(40).

¿Y qué ocurre con los cánceres? Según señala la correspondiente Guía Clínica, internacionalmente el cáncer Cervicouterino está dentro de las tres primeras causas de muerte por cáncer en mujeres, junto con el cáncer de mama y cáncer de pulmón" (41). Como sucede en general con todos los cánceres, se requiere de componentes básicos en el Nivel Terciario de Atención, además de Centros de Quimioterapia y Radioterapia, para darles debido tratamiento. Pero sólo existen 18 Centros Panda en el país — centros de cáncer del adultoque se ubican en los establecimientos de mayor complejidad de la red, lo que significa que hay miles de personas que no tienen, en su ciudad o población, un centro de salud que pueda atenderles. Es más, hay tres regiones en Chile que simplemente no poseen ni uno sólo de dichos centros (III, VI y XI). Lo mismo acontece con la Red de Centros de Radioterapia, que se compone de sólo 10 centros, habiendo cuatro regiones que no poseen ninguno(42). Este tipo de cáncer, por cierto, también tiene restricción por edad, cuestión que 


\section{Tabla 1}

\begin{tabular}{|c|c|}
\hline PATOLOGÍA & CRITERIOS DE INCLUSIÓN/EXCLUSIÓN \\
\hline Accidente cerebro vascular isquémico & Edad: 15 ańos y más \\
\hline Alivio al dolor por cáncer y cuidado paliativo & Lugar de residencia \\
\hline Analgesia en el parto & Lugar de residencia \\
\hline Artrosis de cadera & Edad: 65 años y más \\
\hline Cáncer Cervicouterino & Lugar de residencia y edad \\
\hline Cáncer de mama & Edad: más de 15 años \\
\hline Cáncer de próstata & Edad: más de 15 años \\
\hline Cáncer de testículos & Edad: más de 15 años y lugar de residencia \\
\hline Cáncer de vesícula & Edad: entre 35 y 49 años \\
\hline Cáncer gástrico & Edad: 15 años y más \\
\hline Cardiopatía congénita operable & Edad: menores de 15 años \\
\hline Cataratas & Lugar de residencia \\
\hline Dependencia de alcohol y drogas & Edad: menores de 20 años \\
\hline Depresión & Edad: 15 años y más \\
\hline Desprendimiento de retina & Lugar de residencia \\
\hline Diabetes Mellitus Tipo 1 & Lugar de residencia \\
\hline Dificultad respiratoria recién nacido & Lugar de residencia \\
\hline Displasia broncopulmonar del prematuro & Lugar de residencia \\
\hline Disrrafias espinales & Lugar de residencia \\
\hline Enfermedad de Gaucher & Lugar de residencia \\
\hline Enfermedad pulmonar obstructiva & Lugar de residencia \\
\hline Epilepsia en adultos & Edad y lugar de residencia \\
\hline Epilepsia no refractaria & Edad: hasta 15 años \\
\hline Escoliosis & Edad: menor de 25 años \\
\hline Esquizofrenia & Primera manifestación de la enfermedad y lugar de residencia \\
\hline Estrabismo & Edad: menor de 9 años \\
\hline Fibrosis quística & Lugar de residencia \\
\hline Fisura labiopalatina & Nacimiento después de vigencia decreto \\
\hline Hemofilia & Lugar de residencia \\
\hline Hemorragia cerebral & Lugar de residencia \\
\hline Hernia de Núcleo Pulposo Lumbar & Lugar de residencia \\
\hline
\end{tabular}


Justicia y racionamiento sanitario en el Plan AUGE. Alejandra Zúñiga Fajuri

\begin{tabular}{|c|c|}
\hline Hernias de pared abdominal & Lugar de residencia \\
\hline Hiperplasia benigna de próstata & Lugar de residencia \\
\hline Hipertensión arterial & Edad: 15 años y más \\
\hline Hipoacusia bilateral & Edad: 65 años y más \\
\hline Hipoacusia bilateral del prematuro & Lugar de residencia \\
\hline Infarto Agudo del Miocardio & Lugar de residencia \\
\hline Infección respiratoria aguda & Edad: menor de 5 ańos \\
\hline Insuficiencia renal crónica terminal & Lugar de residencia \\
\hline Linfomas & Lugar de residencia \\
\hline Medicina preventiva & Edad: 15 años y más. Factores de riesgo específicos \\
\hline Neumonía adquirida & Edad: 65 años y más \\
\hline Ortesis (ayuda técnica) & Edad: 65 años y más \\
\hline Politraumatizado & Lugar de residencia \\
\hline Retinopatía diabética & Lugar de residencia \\
\hline Salud oral integral & Edad: por una vez a los 6 ańos \\
\hline Salud oral integral & Edad: por una vez a los 60 años \\
\hline Trastornos de generación del impulso cardíaco & Edad: 15 años y más \\
\hline Tratamiento quirúrgico escoliosis & Edad: menores de 25 años \\
\hline Trauma ocular & Lugar de residencia \\
\hline Traumatismo cráneoencefálico & Lugar de residencia \\
\hline Tumor primario del sistema nervioso central & Edad: 15 años y más \\
\hline Vicios de refracción & Edad: 65 años y más \\
\hline VIH & Lugar de residencia \\
\hline
\end{tabular}

Fuente: Guías Clínicas MINSAL. Tabla elaborada por la autora. 
se produce en el nivel preventivo, pues para ser atendida se debe tener citología cervical positiva o sospecha clínica de cáncer cervicouterino, y el PAP (Papanicolaou) es garantizado por el AUGE sólo a mujeres desde 25 a 64 años(43).

La utilización de los QALY también se evidencia con el cáncer de vesícula que, no obstante ser la principal causa de muerte por cáncer en la mujer chilena y la segunda causa de hospitalización en este mismo grupo poblacional, sólo incluye como beneficiarios de tratamiento a personas de entre los 35 y 49 años. ¿La razón? Un juicio de eficiencia, pues la guía dispone que las restricciones de edad permitirán tratar en su mayoría cánceres incipientes de vesícula "de mejor pronóstico" $(44)^{8}$. No deja de llamar la atención el hecho de que estas enfermedades propiamente femeninas tengan coberturas tan restringidas, pues el AUGE pretendía precisamente reducir las desigualdades sociales y sanitarias generadas en razón del sexo?

Por su parte, el cáncer de testículos tiene tratamiento cubierto entre los 15 y 35 ańos, de modo que fuera de ese rango de edad la persona queda excluida de atención(42). El mismo análisis costo-beneficio se realiza con la epilepsia no refractaria, que excluye al $30 \%$ de la población de pacientes que no responden bien a los medicamentos, esto es, en términos QALY, a enfermos “ineficientes"(45). Esta patología, por cierto, no recibe total cobertura ni aun en los casos de pacientes con buen pronóstico, pues la Guía respectiva contempla, además, exclusión por edad - hasta 15 años-, representando sólo al 70\% de las personas con epilepsia. Por cierto, la propia autoridad reconoce que, si bien se calcula estadísticamente que debieran haber ingresado al nivel de Atención Primaria de Salud (APS) 14.000 niños con problemas neurológicos, sólo lo han hecho alrededor del 25\%. La principal causa de este bajo ingreso radica en que "el recurso neurólogo infantil está presente en un bajo porcentaje de

\footnotetext{
${ }^{8}$ Hay que destacar que poseemos la tasa de mortalidad más alta del mundo en Araucanía Norte con 38,2/100.000 hab. (Departamento de Estadísticas e Información de Salud; 2005).

${ }^{9}$ Sobre los mecanismos para alcanzar mayores grados de igualdad para las mujeres por medio de políticas sanitarias véase Payne Sarah. How can gender equity be addressed through health systems? Policy Brief 12, Copenhagen: World Health Organization, European Observatory on Health Systems and Policies; 2009.
}

las necesidades" y la contrarreferencia a Atención Primaria no cumple en dar atención dentro del plazo establecido $(46)^{10}$.

La Guía de Medicina Preventiva dispone, a su turno, que para ser beneficiario de los exámenes preventivos de cáncer cervicouterino es necesario tener entre 25 a 64 años. Si se quiere acceder al examen de colesterol, se precisan 40 años o más. Sólo a los adultos de más de 65 años se les garantiza una evaluación funcional. Finalmente, la posibilidad de una mamografía, por solo una vez en la vida, se entrega a las mujeres de 50 años(43).

La falta de acceso a las garantías GES en razón del lugar de residencia es, como en el caso de la edad, mayoritaria en el Plan AUGE. Según los datos de la Superintendencia de Salud sobre prestadores de salud por Región, existen en Chile 22 Hospitales tipo 1 y 37 Hospitales tipo 2, lo que evidencia una notable escases de hospitales capaces de asegurar los tratamientos y servicios GES, tanto por la falta de recursos técnicos, como de recursos humanos. Incluso en aquellos hospitales tipo 1 y 2 los especialistas trabajan solamente media jornada, de modo que no tienen la capacidad de brindar la atención a la que la autoridad se había comprometido pues, para ello, sería necesario contar con un sistema de salud dotado del doble de especialistas con jornadas de trabajo completas. Mientras ello no ocurra, los incumplimientos en las garantías AUGE seguirán creciendo.

¿Qué alternativas tenía la autoridad sanitaria al momento de delinear el contenido y los beneficiarios del mínimo sanitario representado por las enfermedades GES-AUGE? Para empezar, se requería trabajar sobre la base de fundamentos teóricos capaces de responder adecuadamente a los "dilemas éticos trágicos", es decir, aquellos que determinan tanto quienes podrán beneficiarse de un cierto tratamiento médico como quienes, en cambio, habrán de morir por falta de atención médica.

\footnotetext{
${ }^{10}$ Los problemas de acceso al sistema por enfermedades neurológicas se agudizan toda vez que el recurso de "especialista neurólogo" es suficiente sólo en el 26\% de los Servicios de Salud, concentrados en su mayoría en la Región Metropolitana (Guía Clínica "Epilepsia no Refractaria en Personas desde 1 ańo y menores de 15 ańos de edad". Santiago de Chile: Minsal; 2005).
} 
Teniendo presente las condiciones de escasez y la demanda por recursos sanitarios siempre en expansión -lo que hace inevitable aplicar alguna forma de racionamiento compatible con la doctrina del suficientismo sanitario-, la autoridad debía, una vez definido un "mínimo sanitario decente" (como las actuales enfermedades AUGE), garantizarlo por igual a todo aquel que lo necesitase, esto es, de modo universal. "Cuando no hay recursos para salvarlos a todos, debemos elegir quienes no serán salvados de una forma que no parezca una preferencia injusta" (47). Una defensa del suficientismo como mejor expresión del igualitarismo sanitario permitía perfilar un cierto contenido de prestaciones como mínimo sanitario, teniendo presente tanto los fines de la equidad como la eficiencia, y asegurando a todos los chilenos con igual necesidad sanitaria la debida atención. Las políticas públicas tienen la obligación de reducir los altos costos sanitarios de la pobreza, esto es, el conocido fenómeno que evidencia las consecuencias que en la salud de las personas se pueden asociar directamente a su condición de pobres(48). El AUGE sigue fallando en esta importante tarea.

\section{Conclusión}

En la construcción de la Reforma Sanitaria AUGE la autoridad ha asumido erróneamente que a la sociedad no le importa cómo se distribuye la suma total de beneficios sanitarios entre sus ciudadanos, lo que suele referirse como "neutralidad distributiva" (21:21-6). En la óptica de los QALY, la mayoría de las Guías Clínicas manifiestan con claridad que buscan otorgar una atención de salud sobre la base de cálculos costo/efectivos que, según se ha mostrado en esta investigación, pueden violentar de distinta forma las promesas que equidad declaradas por la autoridad en su momento.

Las teorías de la justicia sanitaria de tipo igualitaria rechazan la incorporación de criterios de racionamiento que, dando prioridad al principio de eficiencia, desconocen que las instituciones públicas no están autorizadas a discriminar entre personas hasta el punto que ello les signifique la posibilidad de vivir o morir. Al implementarse la Reforma Sanitaria, se debió buscar que los actores encargados de la administración de recursos fueran capaces de concebir respuestas éticamente fundadas frente a los problemas de justicia que se han generado con la ejecución de las GES. Los responsables administrativos deben tener en cuenta otros móviles —además de la eficienciaa la hora de optar por la más justa decisión distributiva. 


\section{Referencias}

1. Beauchamp TL, Childress JF. Principles of Biomedical Ethics. Sixth Edition. New York: Oxford University Press; 2009: 241-243.

2. Rawls J. A Theory of Justice. Oxford: Oxford University Press; 1971: 12.

3. Rawls J. Liberalismo politico. México: Fondo de Cultura Económica; 1995: 39-40.

4. Rawls J. La Justicia como equidad: una reformulación. Barcelona: Paidós; 2002: 73.

5. Rawls J. Lecciones sobre la historia de la filosofía moral. Barcelona: Paidós; 2001:170.

6. Parfit D. Equality and Priority. Ratio 1997; X(3): 204.

7. Casal P. Why Sufficiency Is Not Enough. Ethics 2007; 117: 308.

8. Frankfurt H. Equality as a Moral Ideal. Ethics 1987; 98(1): 21.

9. Broome J. Equality versus Priority: A Useful Distinction. In: Wikler D, et al. (eds.) Fairness and Goodness in Health. Organización Mundial de la Salud (forthcoming).

10. Crisp R. Equality, Priority, and Compassion. Ethics 2003; 113: 752-753.

11. Powers M, Faden R. Social Justice: The Moral Foundations of Public Health and Health Policy. Oxford: Oxford University Press; 2006: 50-79.

12. Brock DW. Priority to the Worse off in Health-Care Resource Prioritization. In: Rhodes R, Battin MP, Silvers A, (eds.) Medicine and Social Justice. Essays on the Distribution of Health Care. OxfordNew York: Oxford University Press; 2002: 371.

13. Dworkin R. Sovereign Virtue. The Theory and Practice of Equality. Londres: Harvard University Press; 2000: 309.

14. Dworkin R. Justice in the Distribution of Health Care. McGill Law Journal 1993; 38(4): 888-889.

15. Zúñiga F. A. Los Sistemas Sanitarios y el AUGE. Acta Bioethica 2007; 13(2): 237-245.

16. Daniels N. Just Health Care. New York: Cambridge University Press; 1985.

17. Daniels N. Just Health. Meeting Health Needs Fairly. Cambridge: Cambridge University Press; 2008: 21-47.

18. Daniels N, Sabin JE. Setting Limits Fairly. New York: Oxford University Press; 2002.

19. Puyol Á. Justícia i Salut. Etica per al Racionament dels Recursos Sanitaris. Barcelona: Bellaterra; 1999: 124-125.

20. Harris J. QALYfying the Value of Life. Journal of Medical Ethics 1987; 13: 117-118.

21. Nord E. Cost-Value Analysis in Health Care. Making Sense out of QALYs. Cambridge: Cambridge University Press; 1999.

22. Nord E, Richardson J, Street A, Kuhse H, Singer P. Who Cares About Cost? Does Economic Analysis Impose or Reflect Social Values? Health Policy 1995; 34.

23. Williams JR, Yeo M. The Ethics of Decentralizing Health Care Priority Setting in Canada. En: Coulter A, Ham C, (eds.) The Global Challenge of Health Care Rationing. Buckingham: Open University Press; 2000.

24. McKie J, Richardson J, Singer P, Kuhse H. The Allocation of Health Care Resources. An Ethical Evaluation of the 'QALY' Approach. Dartmouth Publishing Company, Ashgate Publishing Limited; 1998.

25. Tsuchiya A. QALYs and ageism: philosophical theories and age weighting. Health Economics 2000; 9(1): 65.

26. Harris J. Micro-allocation: deciding between Patients. En: Kuhse H, Singer P, (eds.) A Companion to Bioethics. London: Blackwell Publishing; 2001.

27. Harris J. Justice and Equal Opportunities in Health Care. Bioethics 1999; 13(5): 392.

28. Brock DW. Justice and the ADA: Does Prioritizing and Rationing Health Care Discriminate Against the Disabled? Social Philosophy and Policy 1995; 12(2).

29. Fried Ch. Rights and Health Care. Beyond Equity and Efficiency. New England Journal of Medicine 1975; 293(5). 
30. Dickenson D. Is efficiency ethical? Resource issues in health care. In: Almond B, (ed). Introducing applied ethics. Oxford UK: Blackwell; 1995: 236-237.

31. Elster J. Justicia Local. Barcelona: Gedisa; 1994: 87-89.

32. Waring DR. Medical benefit and the human lottery. An egalitarian approach to patient selection. The Netherlands: Springer; 2004: 187-190.

33. Calabresi \& Bobbitt. Tragic Choice. New York: Norton \& Company; 1978.

34. Guía Clínica. Displasia Broncopulmonar del Prematuro. Santiago de Chile: Minsal; 2005.

35. Guía Clínica. Tratamiento Quirúrgico de Escoliosis en Menores de 25. Santiago de Chile: Minsal; 2005.

36. Informe sobre la Salud en el Mundo 2003: Forjemos un futuro. Nueva York: OMS; 2003: 136-137.

37. Informe sobre la Salud en el Mundo 2000. Mejorar el desempeño de los Sistemas de Salud. Nueva York: OMS; 2000.

38. Vega J, et al. Chile: Socioeconomic differentials and mortality in a middle-income nation. En: Evans, Whitehead, Diderichsen, Bhuiya y Wirth (eds). Challenging Inequities in Health, from ethics to Action. Oxford University Press; 2001.

39. Guía Clínica. Analgesia del Parto. Santiago de Chile: Minsal; 2007.

40. Guía Clínica. Endoprótesis Total de Cadera en personas de 65 años y más con Artrosis de Cadera y Limitación Funcional Severa. Santiago de Chile: Minsal; 2005.

41. Guía Clínica. Cáncer Cervicouterino. Santiago de Chile: Minsal; 2005.

42. Guía Clínica. Cáncer de Testículo en personas de 15 años y más. Santiago de Chile: Minsal; 2005.

43. Guía Clínica. Examen de Medicina Preventiva. Santiago de Chile: Minsal; 2006.

44. Guía Clínica. Colecistectomía preventiva del Cáncer de Vesicula en adultos de 35 a 49 años sintomáticos. Santiago de Chile: Minsal; 2006.

45. Guía Clínica. Epilepsia no Refractaria en Personas desde 1 año y menores de 15 años de edad. Santiago de Chile: Minsal; 2005.

46. Guía Clínica. Epilepsia en el adulto. Santiago de Chile: Minsal; 2007.

47. Harris J. More and better justice. In: Bell JM, Mendus S, (eds.) Philosophy and medical welfare. Cambridge: Cambridge University Press; 1998: 90.

48. Kaplan GA. The Poor Pay More. Poverty's High Cost to Health. Founder Center for Social Epidemiology and Population Health, University of Michigan; 2009.

Recibido: 19 de octubre de 2009

Aceptado: 11 de febreo de 2010 